\title{
Equilibrium shapes of a ferrofluid drop
}

\author{
O. Lavrova*1, V. Polevikov ${ }^{* * 2}$, and L. Tobiska ***1 \\ ${ }^{1}$ Institut für Analysis und Numerik, Otto-von-Guericke-Universität Magdeburg, PF4120, 39016 Magdeburg, Germany \\ 2 Department of Computational Mathematics, Belarusian State University, F. Skaryna Ave. 4, 220050 Minsk, Belarus
}

\begin{abstract}
A numerical solution strategy for calculating equilibrium free surfaces of a ferrofluid drop under the action of uniform magnetic fields is proposed. Based on this strategy, drop shapes of nonlinear magnetisable fluids are obtained numerically in a wide range of field intensities and compared with existing theoretical results.
\end{abstract}

C) 2005 WILEY-VCH Verlag GmbH \& Co. KGaA, Weinheim

\section{Introduction}

The problem for calculating equilibrium free surfaces of a ferrofluid drop is described by a coupled system of the Maxwell's equations and the magnetically augmented Young-Laplace equation [4]. The Maxwell's equations are formulated inside of the bounded fluid domain $\Omega_{1}$ with an a-priori unknown boundary $\Gamma$ and outside in the air domain $\Omega_{2}=\mathbb{R}^{3} \backslash \Omega_{1}$. The boundary position $\Gamma$ will be determined as a solution of the Young-Laplace equation. Both the magnetic field and drop surface have to be found simultaneously. One possible way to handle numerically the coupled problem is to split it into two subproblems: solving the Maxwell's equations for the given drop shape and solving the Young-Laplace equation for the given field distribution on the interface $\Gamma$. Iterations between two subproblems will result in the equilibrium shape. Discretisation of the Maxwell's equations is realised by the coupled boundary-element/finite-element method and for the Young-Laplace equation by the finite-difference method.

\section{Mathematical formulation}

Under the assumption of the axial symmetry for the drop shape we formulate the mathematical model in cylindrical coordinates $(r, z)$. Taking radius $R_{0}$ of the initial circular drop shape as a characteristic length and intensity of the applied field $H_{0}$ as a characteristic field strength we write the two-dimensional Maxwell's equations complemented by the boundary conditions in dimensionless form

$$
\begin{array}{ll}
-\nabla \cdot\left(\mu\left(\left|\nabla u_{1}\right|\right) \nabla u_{1}\right)=0 \quad \text { in } \quad \Omega_{1}, & -\nabla \cdot\left(\nabla u_{2}\right)=0 \quad \text { in } \Omega_{2} ; \\
u_{1}=u_{2}, \quad \mu\left(\left|\nabla u_{1}\right|\right) \frac{\partial u_{1}}{\partial n}=\frac{\partial u_{2}}{\partial n} \quad \text { on } \quad \Gamma, \\
u_{2}=z, \quad(r, z) \rightarrow \infty ; \\
\mu(t)=1+3 \chi \frac{(\operatorname{coth} \gamma t-1 /(\gamma t))}{\gamma t}, \quad \gamma=\frac{3 \chi H_{0}}{M_{s}} .
\end{array}
$$

Here $u_{1}$ and $u_{2}$ denote the scalar potentials in $\Omega_{1}$ and $\Omega_{2} ; \chi$ is the initial susceptibility, $M_{s}$ the magnetic saturation.

We describe the equilibrium shape of the ferrofluid drop by parametric functions $r=r(s), z=z(s)$, where $s$ denotes the arc length of the equilibrium line $\Gamma$. Using the approach in [3] the Young-Laplace equation can be written as

$$
\begin{aligned}
& z^{\prime \prime}=r^{\prime} F, \quad r^{\prime \prime}=-z^{\prime} F, \quad 0<s<1 ; \quad F=f-\frac{z^{\prime}}{r}+C \\
& r(0)=0, z^{\prime}(0)=0, \quad r^{\prime}(1)=0, z(1)=0 \\
& f=-W L\left[\frac{2}{3 \chi} \ln \frac{\sinh (\gamma H)}{\gamma H}+\left(\left(\operatorname{coth} \gamma H-\frac{1}{\gamma H}\right) \frac{H_{n}}{H}\right)^{2}\right], \quad W=\frac{\mu_{0} M_{s}^{2} V^{1 / 3}}{2 \sigma} .
\end{aligned}
$$

The point $s=0$ lies on the $z$ axis and the point $s=1$ on the plane $z=0$. For details of calculating constants $C$ and $L$ see [3]. Here $H=\left|\nabla u_{1}\right|$ is the magnetic field intensity, $H_{n}=\partial u_{1} / \partial n, \mu_{0}$ denotes the magnetic permeability, $V=4 \pi R_{0}^{3} / 3$ the drop volume, $\sigma$ the surface tension coefficient.

* Corresponding author: e-mail: olga.lavrova mathematik.uni-magdeburg.de, Phone: +493916712633 , Fax: +493916718073 ,

** e-mail: polevikov@bsu.by, Phone: +375172095532 , Fax: +375172265548.

*** e-mail: lutz.tobiska@mathematik.uni-magdeburg.de, Phone: +4939167 18650, Fax: +493916718073. 


\section{Discretisation of the problem}

To discretise equations (1)-(3) we use the idea of the coupled collocation boundary-element method (BEM) and the Galerkin finite-element method (FEM), analysed in [5]. We apply the BEM in the unbounded domain $\Omega_{2}$ to fulfill exactly the boundary condition for the magnetic field at the infinity (3). The FEM is used in the bounded domain $\Omega_{1}$, where nonlinearities of the Maxwell's equations occur. We reformulate equations (1)-(3) as the nonlocal boundary value problem for $u_{1}$ in $\bar{\Omega}_{1}$ and $\partial u_{1} / \partial n$ on $\Gamma$. Piecewise linear and piecewise constant functions are used for the approximation of $u_{1}$ and $\partial u_{1} / \partial n$, respectively. The fluid-air interface $\Gamma$ is fixed during the process of solving equations (1)-(3) with a piecewise linear approximation of the interface in the FEM discretisation and a cubic spline approximation in the BEM discretisation being used. The resulting nonlinear discrete system is solved by a fixed-point iteration method with application of the Gaussian elimination method for the linearised systems.

We use a finite-difference scheme of the second order approximation to discretise equations (4)-(5), for details see [3]. Grid adaptation based on the information about the surface curvature is applied [3]. A two-layer iterative scheme is constructed with tridiagonal matrices of the linearised systems.

\section{Numerical results}

The current work extents results of [2], where only linear magnetisable fluids were considered. The linear magnetisation is a reasonable assumption in the region of weak fields [4], whereas nonlinear magnetisation is a necessary requirement for the problem modeling in a wide range of field intensities.

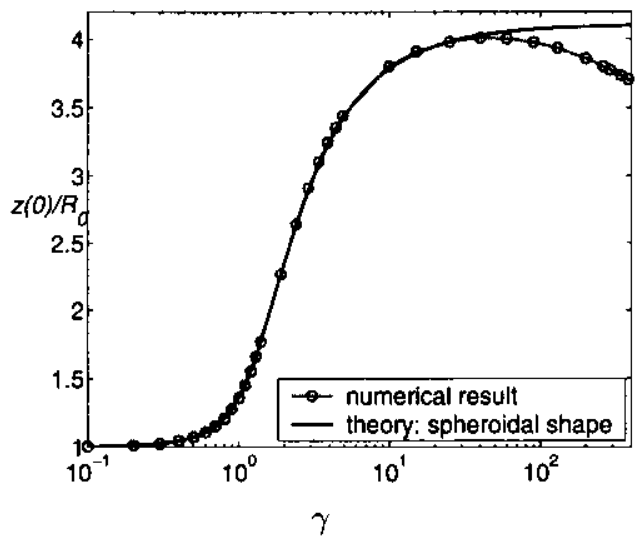

Fig. 1 The dependence of the drop elongation versus the dimensionless magnetic field. Calculations were made for the ferrofluid with parameters $\chi=1.9, W=62$.

If we assume that the drop shape is spheroid then we can determine the drop elongation for every field intensity by applying a theoretical approach, so-called the virial method [1]. The corresponding dependence is drawn by the solid line. The curves with markers present numerical results. From the picture we see that for $\gamma \in[0,50]$ theoretical and numerical results nearly coincide. It follows that for the considered ferrofluid the drop shape is rather close to spheroid in a wide range of field intensities. For $\gamma>50$ the numerical results show a qualitative difference with the theory: a larger applied field produces less elongated shape. Such a behavior of numerical results should be further studied.

Acknowledgements The authors would like to thank the State Sachsen-Anhalt and DFG-Project TO 143/4-2 for supporting the research.

\section{References}

[1] E. Blums, A. Cebers, M. Maiorov, Magnetic fluids (De Gruyter, Berlin, 1997).

[2] O. Lavrova, G. Matthies, V. Polevikov, L. Tobiska, Proc. Appl. Math. Mech. 4, 704-705 (2004).

[3] V. K. Polevikov, Comput. Methods in Applied Math. 4, No. 1, 66-93 (2004).

[4] R. E. Rosensweig, Ferrohydrodynamics (Dover Pubns, New York, 1998).

[5] W. L. Wendland, in Finite element and boundary element techniques from mathematical and engineering point of view, edited by E. Stein (Springer, Wien, 1988), p. 273. 